epidemiology provides evidence of genotypic heterogeneity of multidrug-resistant Pseudomonas aeruginosa serotype 012 outbreak isolates from a pediatric hospital. $J$ Clin Microbiol 1996;34:3226-3229.

5. Schlaifer D, Rixe O. Lost potential in France? Science 1998;279:1431-1432.

Joseph Watine, DrPharmBiol Hôpital Général Rodez, France

\section{Vancomycin-Resistant Enterococci in Hemodialysis Patients Is Related to Intravenous Vancomycin Use}

\section{To the Editor:}

In many hospitals worldwide, there has been an increase in vancomycin-resistant enterococci (VRE) colonization and infection and in the use of intravenous vancomycin. To study the relation of parenteral vancomycin use to VRE colonization and infection, we prospectively examined our hemodialysis (HD) patient population, because vancomycin is frequently used for prophylaxis or treatment of staphylococcal infections in HD patients. ${ }^{1-5}$

The target population of the surveillance screening included all clinically stable $\mathrm{HD}$ patients dialyzed during six daytime shifts at the outpatient dialysis unit. Patients screened had been undergoing routine HD for a minimum of 3 months. Patients were excluded from analysis if their age was $<18$ years, if there had been a hospital admission in the prior month, if they did not consent to be studied, or if there was a known enterococcal infection in the previous year.

Rayon-tipped rectal swabs were obtained from 111 consenting HD patients immediately prior to dialysis treatment and were transported promptly to the microbiology laboratory in $\mathrm{BBL}$ culturette transport media (BBL Microbiology Systems, Cockeysville, MD). Specimens were planted promptly onto bile-esculin agar (BBL) and incubated at $35^{\circ} \mathrm{C}$ for up to 48 hours before being discarded as negative. Colonies developing a black to brown color were identified as enterococci, based on the pyrrolidonyl arylamidase disk test (PML Microbiologicals, Tualatin, $O R$ ) and tolerance to $6.5 \% \mathrm{NaCl}$ (BBL). Enterococcal isolates were tested for susceptibility to vancomycin by the standardized disk-diffusion procedure. A suspension of organisms equivalent to a McFarland 0.5 barium sulfate turbidity standard was prepared in trypticase soy broth (BBL) and swabbed onto the surface of a Mueller-Hinton agar plate (BBL). After the application of antibiotic disks, the plates were incubated at $35^{\circ} \mathrm{C}$ in ambient air for 24 hours. Zone size interpretations suggested by the National Committee for Clinical Laboratory Standards (December 1993) were used to determine the susceptibility of isolates to vancomycin and teicoplanin.

Patients found to be VRE-positive at initial screening did not receive any specific treatment. Charts were reviewed for demographic information, cause of end-stage renal disease, number of hospital days, use of intravenous or oral vancomycin, and intensive-care unit (ICU) hospitalizations in the previous year. The initial cohort was followed for 1 year, during which all microbiological isolates and any evidence of relevant infection were evaluated. An investigator summarized each patient event during the year with regard to the culture results and clinical disease episodes. Isolation of VRE from a normally sterile site, in association with clinical symptoms and signs, was considered to represent clinically relevant infections.

Statistical analysis used Student's unpaired $t$ test for continuous variables and Fisher's Exact Test for discrete variables. All results are reported as mean \pm standard deviation. A $P$ of $<.05$ was considered significant.

A total of 111 patients (63 male) with a mean age of $59.9 \pm 11.3$ (range,
20-85) years had VRE. The mean duration of dialysis treatment was $23.2 \pm 10.7$ months.

Enterococcus was isolated from $91(82 \%)$ of 111 patients. Ten patients (9\%) had isolates that were resistant to vancomycin; 3 of these 10 isolates also were resistant to teicoplanin. Various factors were analyzed as possible predictors of VRE colonization (Table). The only statistically significant difference between VRE-positive and VRE-negative patients was in the volume of intravenous vancomycin exposure in the year prior to study $(4.4 \pm 5.9 \mathrm{~g}$ compared to $1.4 \pm 2.5 \mathrm{~g}$, respectively; $P=.003$ ). Of note, risk factors for VRE colonization found in studies of nonuremic populations (number of hospital days, number of ICU days, and use of multiple antibiotics) were not found to be predictors in this hemodialysis population.

In the year of follow-up, clinically relevant VRE infection developed in 3 (2.7\%) of the 111 patients. During the year, there were 24 deaths, 6 patients received transplants, and 3 had incomplete follow-up. In total, there were 1,144 patient-months observed, yielding an incidence rate of 0.03 cases per patient-month. VRE infections included a sacral osteomyelitis in 1 patient, bacteremia in a second, and a peritoneal dialysis-associated peritonitis in a third. Of these 3 patients, 2 initially were VRE-negative and 1 was VRE-positive. Thus, $2(1.9 \%)$ of the 101 patients who were VRE-negative subsequently developed a VRE infection, compared to $1(10 \%)$ of 10 patients initially VRE-positive (not significant).

In summary, we found VRE rectal carriage in $9 \%$ of stable HD patients, with prior intravenous van-

TABLE

Rectal Carriage of Enterocci in Hemodialysis Patients

\begin{tabular}{lccl}
\hline & VRE & No VRE & $\boldsymbol{P}$ \\
\hline Subjects & 10 & 101 & - \\
Age (y) & $61.7 \pm 12.2$ & $59.4 \pm 11.2$ & NS \\
Male:female & $3: 7$ & $60: 41$ & NS \\
Days hospitalized in the past year & $15.8 \pm 25.9$ & $15.6 \pm 22.2$ & NS \\
IV vancomycin in the past year (g) & $4.4 \pm 5.9$ & $1.4 \pm 2.5$ & .003 \\
Total antibiotic doses in the past year (no. of doses) & $9.1 \pm 8.3$ & $8.9 \pm 8.0$ & NS \\
ICU hospital days in the past year & $1.9 \pm 7.5$ & $1.1 \pm 2.4$ & NS \\
Vascular catheter-days in the past year & $4.8 \pm 5.3$ & $4.1 \pm 5.5$ & NS
\end{tabular}

Abbreviations: $1 C U$, intensive-care unit; IV, intravenous; NS, not significant; VRE, vancomycin-resistant enterococci. 
comycin a significant predictor for VRE carriage. Interestingly, neither the number of hospital days nor prior ICU admissions were found to be significant risk factors for VRE colonization. There were three infections (2.7\% of subjects) with VRE, but there was no relation between prior VRE carriage and subsequent risk for clinically relevant infection.

Although some studies have reported a low prevalence of VRE in patients with renal failure, we found that VRE colonization was not uncommon among hemodialysis patients. Until more is known about VRE colonization, we recommend that intravenous vancomycin be reserved for documented serious methicillin- resistant staphylococcal infections. Vancomycin should not be used for empirical therapy and should not be used to treat methicillin-resistant Staphylococcus aureus colonization.

We conclude that intravenous, not oral, vancomycin use predisposes to VRE colonization in HD patients.

\section{REFERENCES}

1. Moellering RC. Emergence of Enterococcus as a significant pathogen. Clin Infect Dis 1992;14:1173-1178.

2. Edmond MB, Ober JF, Weinbaum DL. Vancomycin-resistant Enterococcus faecium bacteremia: risk factors for infection. Clin Infect Dis 1995;20:1126-1133.

3. Jordens JZ, Bates J, Griffiths DT. Faecal carriage and nosocomial spread of vancomycinresistant Enterococcus faecium. J Antimicrob Chemother 1994;34:515-528.
4. Tornieporth NG, Roberts RB, John J, Hafner A, Riley LW. Risk factors associated with vancomycin-resistant Enterococcus faecium infection or colonization in 145 matched case patients and control patients. Clin Infect Dis 1996;23:762-772.

5. Fraise AP. The treatment and control of vancomycin-resistant enterococci. $J$ Antimicrob Chemother 1996;38:753-756.

Steven Fishbane, MD

Burke A. Cunha, MD

Sanjeev K. Mittal, MD John Ruggian, MD

Kevin Shea, MD

Paul E. Schoch, PhD

Winthrop-University Hospital Mineola, New York

State University of New York School of Medicine

Stony Brook, New York

\section{Inactivation of Duck Hepatitis B Virus by Hydrogen Peroxide Gas Plasma Sterilization}

\section{Gina Pugliese, RN, MS Martin S. Favero, $\mathbf{P h D}$}

Human hepatitis B virus (HBV) is an important cause of nosocomial infections and can be transmitted by contaminated instruments. However, tests of the efficacy of sterilization of materials and equipment contaminated by $\mathrm{HBV}$ are difficult to perform because the virus cannot be grown in the laboratory. As a result, investigators studying the inactivation of $\mathrm{HBV}$ have begun to use other viruses in the hepadna group of viruses as surrogates for HBV. Vickery and coinvestigators from the University of Sidney in Australia conducted a study where the objective was to evaluate the capability of a low- temperature, hydrogen peroxide gas plasma sterilizer (Sterrad, Advanced Sterilization Products, Irvine, CA) to inactivate duck hepatitis $B$ virus (DHBV).

In laboratory efficacy studies using DHBV dried on to glass filter carriers and exposed to one half of the hydrogen peroxide gas plasma sterilization process, there was a $10^{7}$ or greater decrease in the viral titer, with no infectivity detected on the carriers after treatment. In-use studies were performed using a laparoscope that was experimentally contaminated with DHBV to mimic the possible transmission of infection between successive patients. Following exposure to the hydrogen peroxide gas plasma sterilization process, no transmission of DHBV infection from the laparoscope occurred, despite visually obvious soiling with blood $(\mathrm{N}=8)$, whereas the transmission rate for the unprocessed laparoscope (positive control) was 100\% $(26 / 26)$, and that for instruments after a water wash was $63 \%(7 / 11)$. The authors concluded that the hydrogen gas plasma sterilization process completely inactivates DHBV, a representative of the hepadna group of viruses.

FROM: Vickery K, Deva AK, Zou J, Kumaradeva P, Bissett L, Cossart YE. Inactivation of duck hepatitis $B$ virus by a hydrogen peroxide gas plasma sterilization system: laboratory and "in use" testing. J Hosp Infect 1999;41:317-322. 